\title{
Influence of temperature on microstructure, structural and ferroelectricity evolution properties with nano and micrometer grain size in multiferroic $\mathrm{HoMnO}_{3}$ ceramics
}

\begin{abstract}
The influence of temperature on microstructural, structural and ferroelectric evolution properties of multiferroic holmium manganese oxide $\left(\mathrm{HoMnO}_{3}\right)$ ceramics were investigated. $\mathrm{HoMnO}_{3}$ ceramics were synthesized using a mechanochemical reaction of $\mathrm{Ho}_{2} \mathrm{O}_{3}$ and $\mathrm{Mn}_{2} \mathrm{O}_{3}$ powders in a high energy ball milling machine. The powder were sintered from 600 to $1250{ }^{\circ} \mathrm{C}$ with $50{ }^{\circ} \mathrm{C}$ increments. The results shows the microstructural, structural and ferroelectric hysteresis loop were observed to be dependent on sintering temperatures. The XRD characterization suggests an improvement of crystallinity with increasing sintering temperature. The hexagonal $\mathrm{HoMnO}_{3}$ were observed at temperature $\geq 1200{ }^{\circ} \mathrm{C}$ with the grain size of around $1600 \mathrm{~nm}$. SEM micrographs showed larger grain size as the sintering temperature increased. The SEM results revealed a transformation of crystal structure occurs from orthorhombic to hexagonal at larger grain size regime. Polarization $P$-electric field $E$ ferroelectric properties were observed to be enhanced with the increase of grain size through sintering temperature. The ferroelectric behavior was observed to change with the change of microstructure along with the structure transformation from orthorhombic to hexagonal. A complete systematic studies of the micron-nanometer grain size on microstructure-properties evolution of $\mathrm{HoMnO}_{3}$ multiferrroic ceramics is higlighted.
\end{abstract}

Keyword: Temperature; Microstructure; Structural; Ferroelectricity evolution properties; Nano; Micrometer; Multiferroic $\mathrm{HoMnO}_{3}$; Holmium manganese oxide ceramic 